\title{
Bootstrapping User-Defined Body Tapping Recognition with Offline-Learned Probabilistic Representation
}

\author{
Xiang 'Anthony' Chen ${ }^{1,2}$ * Yang Li ${ }^{1}$ \\ Google Research ${ }^{1}$ \\ Carnegie Mellon University ${ }^{2}$ \\ \{xiangchen, yangli\}@acm.org
}

\begin{abstract}
To address the increasing functionality (or information) overload of smartphones, prior research has explored a variety of methods to extend the input vocabulary of mobile devices. In particular, body tapping has been previously proposed as a technique that allows the user to quickly access a target functionality by simply tapping at a specific location of the body with a smartphone. Though compelling, prior work often fell short in enabling users' unconstrained tapping locations or behaviors. To address this problem, we developed a novel recognition method that combines both offline-before the system sees any user-defined gestures - and online learning to reliably recognize arbitrary, user-defined body tapping gestures, only using a smartphone's built-in sensors. Our experiment indicates that our method significantly outperforms baseline approaches in several usage conditions. In particular, provided only with a single sample per location, our accuracy is $30.8 \%$ over an SVM baseline and $24.8 \%$ over a template matching method. Based on these findings, we discuss how our approach can be generalized to other user-defined gesture problems.
\end{abstract}

\section{Author Keywords}

Motion gesture recognition; on-body interaction; templatebased recognition; KL divergence; user-defined gestures.

\section{ACM Classification Keywords}

H.5.2 [User Interfaces]: Interaction styles. I.5.2 [Pattern recognition]: Design methodology.

\section{INTRODUCTION AND RELATED WORK}

Mobile devices have become increasingly overloaded with functionalities and data-average users had 119 apps on their device as reported by Apple on WWDC 2015, all of which they had to access through a small touchscreen. To expand the input bandwidth of a mobile device, prior work has attempted to enable the user's body-that is always available - as an extended input space of the device [1, 3, $4,10]$. In particular, a body-tapping gesture allows users to quickly access a target functionality by simply tapping a

Permission to make digital or hard copies of all or part of this work for personal or classroom use is granted without fee provided that copies are not made or distributed for profit or commercial advantage and that copies bear this notice and the full citation on the first page. Copyrights for components of this work owned by others than ACM must be honored. Abstracting with credit is permitted. To copy otherwise, or republish, to post on servers or to redistribute to lists, requires prior specific permission and/or a fee. Request permissions from Permissions@acm.org.

UIST 2016, October 16-19, 2016, Tokyo, Japan

(C) 2016 ACM. ISBN 978-1-4503-4189-9/16/10 \$15.00

DOI: http://dx.doi.org/10.1145/2984511.2984541

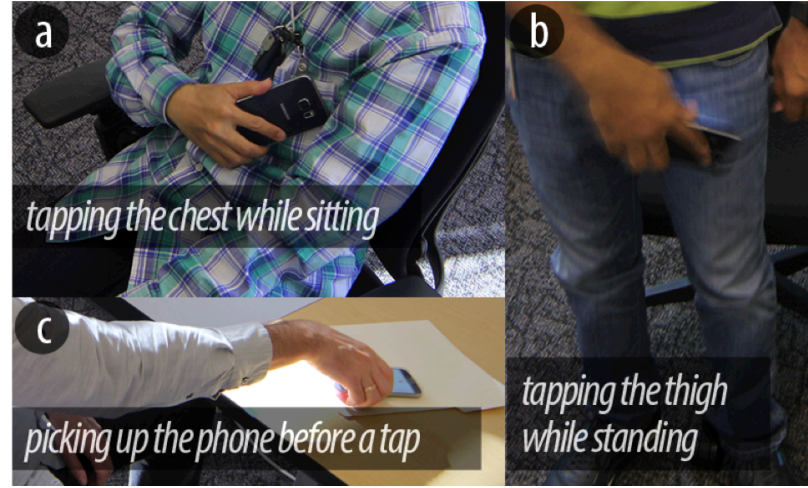

Figure 1. Our method can robustly recognize user-defined, unconstrained body tapping behaviors with a handheld device, in several body postures and initial device placements.

specific part of their body with a handheld device, e.g., tapping the wrist to bring up the calendar app, the belly for searching nearby restaurants or the knee to obtain navigational information [3]. Such body tapping gestures have shown great potential in extending the input capacity of a mobile device.

However, prior work is fairly limited in realizing these body-tapping gestures, primarily due to the difficulty of recognizing a wide range of possible locations users might tap on their body. As such, prior systems often fell short as proofs of concept that required instrumenting additional sensors, e.g., RFID or motion capture systems [3]. To use commodity sensors, prior systems had to impose artificial constraints on the user, e.g., the user has to start with a fixed initial position for each tap and to position the device on an imaginary plane in front of the body [4]. Our goal is to develop a recognition method for user-defined body tapping gestures that can be performed in an unconstrained manner (see Figure 1). We also decided to only rely on built-in sensors for easy deployment on mobile devices.

There are two typical settings for gesture recognition. When the target gestures are predetermined, e.g., alphabetic gestures, one can create a robust recognizer with sufficient training data of these gestures offline before the system is deployed to the user. However, body-tapping gestures can vary greatly across users depending on the specific body locations a user prefers or feels comfortable with tapping. In addition, body differences such as heights and weights

\footnotetext{
* The work was done during an internship at Google Research.
} 
can also have a significant effect on tapping gestures. As a result, offline learning is not entirely feasible for recognizing unconstrained body-tapping gestures.

To recognize user-specific gestures, it is necessary to create a gesture recognizer online as the user provides desired gestures at runtime. To this end, template matching has become a popular approach for user-defined motion gesture recognition (e.g., [6, 7]). It recognizes an unknown gesture by matching it against a set of template gestures provided by the user on the fly. However, this approach suffers when there are few templates available-users are generally unwilling to or incapable of providing sufficient templates at runtime. With insufficient templates, it is difficult for the recognizer to capture the great variance in tapping behaviors such as different body postures, e.g., sitting versus standing (see Figure 1). Furthermore, how the user initiates a tap - the initial placement of the device such as in-hand versus being picked up from a table-also matters because it results in different motion trajectories in addition to the destination of a tap, i.e., the target body location.

In this paper, we combine both offline statistical machine learning and online template matching for body tapping gesture recognition (see Figure 2). While traditional machine learning methods have combined offline and online models, e.g., personalization in speech recognition, the models in these two stages mostly deal with the same target vocabulary. In contrast, our offline and online gesture vocabulary sets can be different. The offline gesture positions are predetermined while the online ones are arbitrarily defined by the user on the fly.

To address these challenges, we learn a representation of body tap gestures offline and then use the representation for template matching of user-defined gestures online, which is different from previous methods in gesture recognition. In particular, our offline learning trains a classifier for recognizing a set of predefined body tapping gestures that are targeted at representative body locations (see Figure 3a). Because a body-tapping gesture is tied to a specific location on the body, we use "gesture" and "location" interchangeably in this paper. In the online stage, for each body-tapping gesture, which is either a user-provided template or an unknown gesture, we featurize it as the probability distribution over the predefined gestures outputted by the offline classifier. We then conduct template matching between gestures based on the similarity of their probabilistic representations as given by $\mathrm{KL}$ Divergence [8]. Our evaluation indicates that our approach significantly outperforms baseline methods especially when there are few templates per body location.

\section{RECOGNITIZING BODY-TAPPING GESTURES}

There are two steps in recognizing a body tapping gesture. First is to detect if a body tapping has occurred and second is to determine where the user has tapped on the body to trigger location-specific functionality. We achieve both steps using built-in sensors on a smartphone.

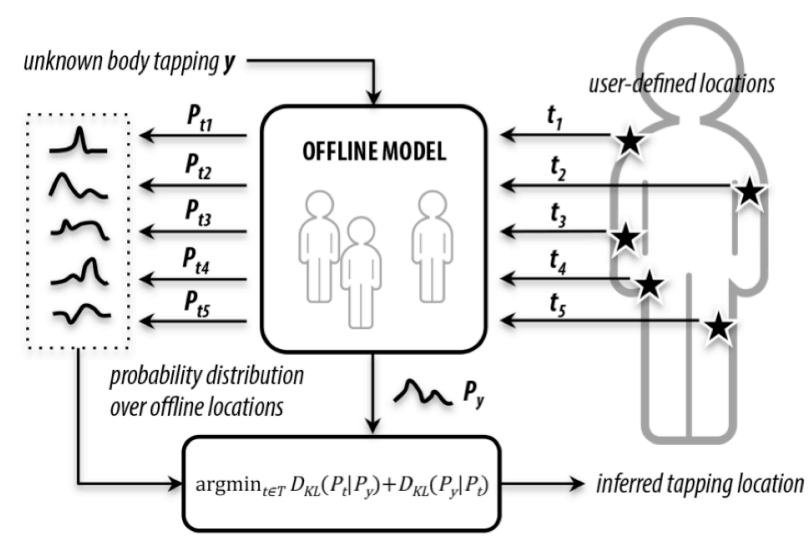

Figure 2. Our contribution is a pipeline of robust recognition of user-defined body tapping locations by combining an offline model (which only includes predefined body locations) and an online template matching method based on KL-divergence.

\section{Motion Sensing and Segmentation}

One challenge in motion gesture recognition is to determine the portion of continuous motion input pertinent to the gesture so that it can be fed to a classifier. Prior work requires the user to explicitly signal the system (e.g., pressing and holding a physical button) preceding a body tapping gesture [4]. To avoid this constraint, we employ a smartphone's proximity sensor to automatically detect the occurrence of a body tap. The proximity sensor is typically located at the top of the device for detecting the phone approaching the ear during a phone call so that the touchscreen can be turned off. When tapping the body with a smartphone's top front part, the proximity sensor immediately detects the physical contact between the body and the device. We found this simple heuristic works reliably well, with very few false positives and negatives as observed in our studies.

One constraint of this approach, though, is that users need to tap the body with the area above the phone's screen where the sensor is located. However, in our study we did not observe participants having any difficulty learning or performing this specific way of tapping.

Having identified when a tapping takes place, we then use the motion sensor readings within a temporal window of a detected body tap for recognizing body-tapping locations. Empirically, we chose to look at a one-second window of motion sensor input-from $750 \mathrm{~ms}$ before to $250 \mathrm{~ms}$ after the time of contact - for classification. We chose to focus on the gyroscope readings in our current implementation, which tends to be most discriminative amongst all the builtin motion sensors. Our feature selection with an initial data set showed that the accuracy of gyroscope is around $92 \%$, accelerometer $88 \%$, and combining both $90 \%$.

For gyroscope, we feed a 1-second time series into the machine learning model. Sampling the 3 axes of the sensor at $200 \mathrm{~Hz}$ leads to a 600 -dimensional vector. We then downsample the data into 10 time buckets for each axis (thus $10 \mathrm{~Hz}$ ), which results in $3 \times 10=30$ features. 
While it is possible to explore other built-in sensors (e.g., touch, camera) with better features to boost the recognition, we did not intend for such a comprehensive exploration; rather, the focus of our contribution is to combine offline and online learning for motion gesture recognition, which is independent of the choice of sensors. In general, our oneline+offline approach does not preclude the use of additional features from other types of sensors.

\section{Offline Learning for Representative Body Locations}

User-defined body-tapping gestures are difficult to determine in advance. However, we hypothesize that we can acquire a general feature representation by learning an offline classification model for a set of predefined tapping gestures targeted at representative body locations. At this offline stage, we can ask a few participants to contribute sufficient training data that would otherwise be difficult to acquire online. We then train the model using an off-theshelf machine learning method, e.g., an SVM. The outcome of the offline learning is a model that outputs a probability distribution over a set of predefined gestures (locations), $R$, given the sensor readings.

\section{Online Template Matching for User-Defined Locations}

At runtime, a user starts by defining a set of body locations for activating each target functionality. To do so, the user performs exemplar gestures by tapping each location, which are recorded by the system as templates. This set of user-defined gestures, $T$, can be different from the predefined gestures, $R$.

To recognize the location of a body tapping, we first feed the motion input of the tap into the offline model. Although the model is trained for a different set of gestures $R$, the outputted probability distribution is still indicative as it characterizes how each predefined gesture in $R$ resembles the unknown tapping gesture. As a result, we can represent each user-specific tap gesture as a probability distribution over the set of gestures predefined in the offline stage, i.e., the probability distribution $P_{y}$ is for an unknown tapping gesture $y$, and $P_{t}$ for a template $t$. We then recognize $y$ as the template $t_{y}$ that has the most similar probabilistic representation:
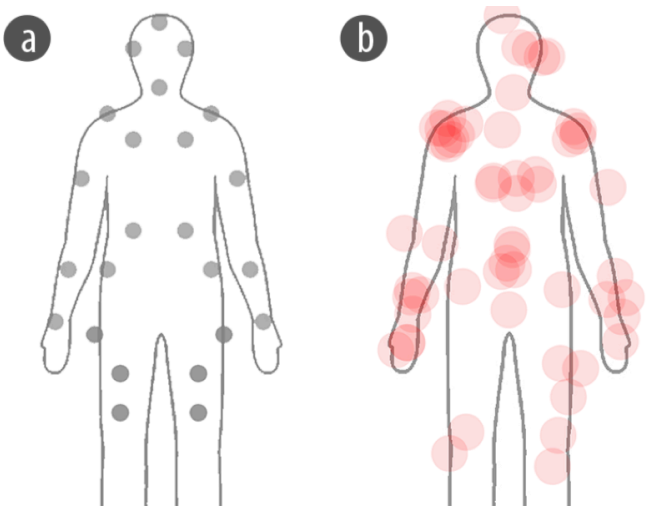

Figure 3. The set of predefined body locations used for training the offline model (a), and the set of body locations selected by participants for the online template matching (b).

$$
t_{y}=\operatorname{argmin}_{t \in T} D_{K L}\left(P_{t} \mid P_{y}\right)+D_{K L}\left(P_{y} \mid P_{t}\right)
$$

where $D_{K L}\left(P_{t} \mid P_{y}\right)$ and $D_{K L}\left(P_{y} \mid P_{t}\right)$ are the Kullback-Leibler (KL) Divergences of $P_{y}$ and $P_{t}$ from both directions (since KL Divergence is asymmetric). KL Divergence is typically used to measure the distance (or dissimilarity) between two probability distributions [8]. Thus, the probability distribution over the set of predefined gestures is used here essentially as the feature representation for a user-specific gesture while the KL Divergence is the distance metric.

\section{EXPERIMENTS}

We hypothesize that our method can significantly improve the recognition of user-defined tapping gestures on arbitrary body locations, even with only few templates available. We here report our experiment to validate this hypothesis.

\section{Data Collection}

We recruited 12 participants from an IT company, 3 female and 9 male, aged 21 to 52 , height $5^{\prime} 3^{\prime \prime}$ to 6 ', weight 100 to $250 \mathrm{lb}$, and 2 left-handed. Our data collection system was running on a Samsung Galaxy S6 Phone with a built-in proximity sensor and gyroscope (sampling rate $200 \mathrm{~Hz}$ ).

We collected two datasets, one for offline and the other for online learning, from each participant with a within-subject design. To simulate a realistic scenario where offline and online data often come from different users, in our analyses, we picked one user's offline data to train our offline model to bootstrap the recognition of a different user's online dataset. To avoid constraining a user with predefined locations, we first collected the online dataset where each user could freely create their own body-tapping gestures. We then collected the offline dataset by asking them to tap at a set of predefined body locations in an instructed way.

\section{Collecting User-Defined Body Tapping Gestures}

In the first part of the experiment, we asked participants to come up with five on-body locations that they prefer to tap with a phone. In our pilot study, we found that participants had trouble coming up with more than five body locations for tapping, due to their actual needs, acceptance [10] and memorability. Thus we felt that five is a reasonable number to ask from the participants. Despite such practical concerns, it is possible to study the resolution upper bound of our approach by overloading tapping locations around a given body part, which we leave as future work.

To help participants memorize their selected on-body locations, we displayed a simple body figure on the phone's screen to let the participants mark and recall their desired locations (see Figure 3b).

Participants were then asked to tap at each self-selected location for 20 times under three different conditions (see Table 1). Conditions were designed to vary participants' tapping behaviors, e.g., with different postures (sitting or standing) and initial device placements (in-hand in front of 
the chest or off-hand on the table). For example, in Condition 2, participants were asked to pick up the phone from the table, tap at a body location and then put it back on the table while remaining seated. Admittedly, it is not possible to include all the postures and device initial placements in one study. Our goal of including these conditions is to push the boundary and test if our approach can handle situations that go beyond a fixed usage setting.

\begin{tabular}{|c|c|c|}
\hline CONDITION & POSTURE & INITIAL DEVICE PLACEMENT \\
\hline 1 & Sitting & In-hand, in front of chest \\
\hline 2 & Sitting & 0ff-hand, on the table \\
\hline 3 & Standing & In-hand, in front of chest \\
\hline
\end{tabular}

Table 1. Posture $\&$ initial device placements for tapping at user-selected body locations. The offline model (trained in Condition 1 only) can handle online data of all 3 conditions.

The order of these conditions was counter-balanced across participants. Participants were instructed to hold the phone normally in the portrait mode for a tap to be detected with the proximity sensor. They were free to use whichever hand they preferred. We did not ask participants to constrain their poses or movements. For example, for tapping at the arm, the participants could either tap it while keeping the arm lowered, or raised, or at any position in between.

For each condition, the target body locations were randomized to avoid temporarily similar tapping patterns. For each trial, the on-screen body figure prompted participants with one of the five locations they selected. Once a tap was registered, the system waited for 1 second before automatically advancing to the next trial. In total, we collected $20 \times 5 \times 3=300$ samples from each participant, which constituted the online dataset for the participant.

\section{Collecting Predefined Body Tapping Gestures}

In the second part of the experiment, we collected data from participants for tapping at a fixed set of 24 body locations (see Figure 3a). We chose these representative locations based on whether tapping on them is physically feasible as well as socially appropriate [10]. They provided a basic coverage of body locations for the offline model.

In this session, participants were asked to hold the phone with alternate hands. For each handedness, there are 21 valid locations, as the upper arm, forearm and wrist on the same side of the holding hand become unreachable. We hypothesize that an offline model does not have to exhaust all tapping behaviors. Thus, we intentionally reduced the conditions previously used for collecting the online data. This allows us to examine how our approach performs when certain conditions that occur online are not encountered at the offline stage. For initial device placements, participants were asked to always start with the phone in hand and in front of the chest. For postures, participants first tapped through 21 reachable locations while sitting. Then they stood up and redid the 6 lowerbody locations.

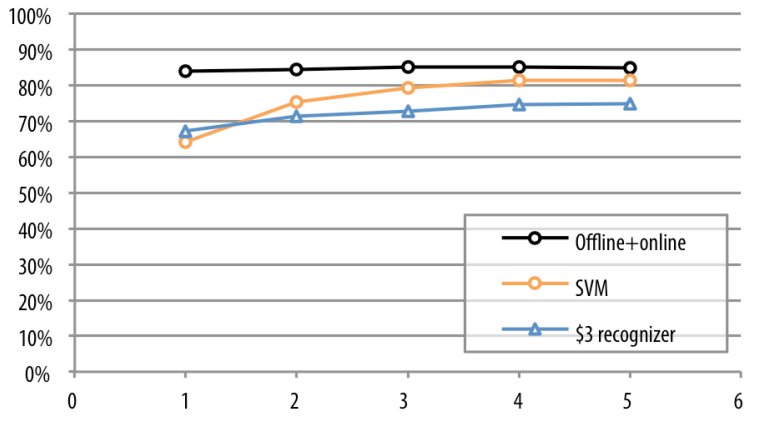

Figure 4. Our method (offline+online) significantly outperforms the two baseline methods.

Participants went through all the $21+6$ taps for 5 times; then they were asked to switch hands. This process was repeated 4 times with the order of handedness counter-balanced across participants. In total, we collected $5 \times(21+6) \times 4=540$ samples from each participant, which formed the offline dataset for the participant.

\section{Experimental Analyses \& Results}

Based on these datasets, we ran our experiment iteratively by learning a model using one user's offline dataset to bootstrap the recognition of the online dataset from each of the other 11 users. Thus, the offline learning was repeated for 12 times, where each offline model was used for bootstrapping the recognition of 11 online datasets. We obtained the overall performance by aggregating results across all 12 rounds.

For an offline dataset, we labeled each sample with its predefine location, body posture and handedness, which resulted in $(21+6) \times 2=54$ distinct labels. We trained an SVM (with a polynomial kernel) to classify a body tapping gesture-given a motion input, the model generates a probability distribution of a 54-element vector. For an online dataset, we randomly selected 5 samples for each of the 5 user-defined locations as templates. Note that these templates might have come from any of the three conditions (see Table 1), which simulated the uncertainty of users' unconstrained tapping behaviors.

We compared our technique with two baseline methods: a statistical machine learning approach-SVM-and a template-based method-the $\$ 3$ gesture recognizer [5]. The gyroscope reading consists of a sequence of (gx, gy, gz) tuples. We preprocessed the data by smoothening it using a Hanning window [5] of size 20 (1/10 of the sampling rate), and then resampled the data into 10 buckets, i.e., $3 \times 10=30$ features for both the SVM and the $\$ 3$ recognizer. We first fed a number of templates into each of the three methods and then tested their performance by recognizing the rest of the online dataset. We repeated this process 10 times.

Overall, our approach (offline+online) consistently outperformed the two baseline methods (Figure 4). The accuracy gain was especially significant when there were few templates available. When there was one template per location, our method achieved $84 \%$ accuracy in recognizing 
unconstrained body-tapping, 30.8\% higher than the SVM baseline and $24.8 \%$ higher than $\$ 3$ recognizer.

We found body locations that are spatially adjacent tend to confuse with each other, e.g., shoulder and collarbone (see Figure 5). However, users tend to pick locations that are distinct enough for both memorability and ease to acquire (Figure 6). As a result, different online gestures often result in distinct representations in our high-dimensional probabilistic feature space. In addition, we consider a 1second motion window (including approaching trajectory) rather than only the moment of impact, which gives additional discriminative power.

\section{DISCUSSION AND CONCLUSION}

The offline model provides an effective representation of body tapping gestures that leads to significant improvement in recognition accuracy during online template matching. This probabilistic representation of gestures can capture the variance that is not sufficiently manifested through a small number of templates provided by the user. Furthermore, the offline model that we learned based on one user's data can bootstrap 11 different users' online template matching, which indicates that our offline-learned representation generalizes well across users.

The results also indicated that the baseline methods, especially the SVM, quickly improved as the number of templates increased. Future work could explore optimally switching methods as users' data accumulates.

Our experiment also revealed users' preferences over tapping on the body. Figure $3 \mathrm{~b}$ shows most people seemed to prefer tapping on their shoulders and forearms. This implies adding prior probabilities over these locations may improve accuracy. Given the various user-defined tapping locations, future work could also formally whether some locations are easier to recognize than the others.

Due to the scope of our study, we only tested static postures; future work could focus on recognition on the go, e.g., walking, running, or in a moving vehicle.

Our approach can be potentially generalized beyond body tapping for other gesture recognition problems, and perhaps using other types of sensors. The essence is to acquire a model for a set of typical gestures in the space, which is easily achievable offline. We then use the offline model to acquire a probabilistic representation of user-defined gestures, which captures how these predefined typical gestures resemble a user-defined one. This brings additional information into the online template matching process that deals with gestures unseen offline. Our approach is easy to apply and different from traditional feature selection techniques or semi-supervised learning that are often concerned with the same set of targets both online and offline. The applicability of our technique is not restricted to SVM or motion sensors. We can use any machine learning method with any features for the offline model, which we plan to investigate in the future.
Our method resonates well with increasingly popular deep learning methods, which are often about learning effective feature representations. These methods typically require a significant amount of data to learn good feature embedding, e.g., unsupervised methods such as auto encoders [2]. In contrast, our method uses a supervised shallow model that requires less data.

Our main contribution is a novel approach for recognizing body-tapping gestures, which uses an offline-learned model to bootstrap online template matching for user-defined gestures. Our approach contributes a novel way for template matching by representing each user-defined gesture as a probability distribution over a set of predefined gestures, and then measuring the similarity between gestures based on their KL Divergence. Our approach significantly outperforms two baselines and can be potentially applied to other gesture recognition problems.

\section{REFERENCES}

1. Ängeslevä J., Oakley I., Hughes S., and O’Modhrain S. 2003. Body Mnemonics Portable device interaction design concept. UIST '03, 2-5.

2. Bengio Y., Yao L., Alain G., and Vincent P. Generalized denoising auto-encoders as generative models. 2013. In Advances in Neural Information Processing Systems. 899-907.

3. Chen X. A., Marquardt N., Tang A., Boring S., Greenberg S. 2012. Extending a mobile device's interaction space through body-centric interaction. MobileHCI '12, 151-160.

4. Guerreiro T., Gamboa R., Jorge J. 2009. Mnemonical body shortcuts for interacting with mobile devices. In Gesture-Based Human-Computer Interaction and Simulation. 261-271. Springer Berlin Heidelberg.

5. Kay S. M. 2013. Fundamentals of Statistical Signal Processing: Practical Algorithm Development. Vol. 3. Pearson Education.

6. Kratz S. and Rohs M. 2010. A \$3 gesture recognizer: simple gesture recognition for devices equipped with 3D acceleration sensors. IUI '10, 341-344.

7. Kratz S. and Rohs M. 2011. Protractor3D: a closedform solution to rotation-invariant 3D gestures. IUI '11, 371-374.

8. Kullback S. and Leibler R. A. 1951. On information and sufficiency. The annals of mathematical statistics. 7986.

9. Ouyang T. and Li Y. Bootstrapping personal gesture shortcuts with the wisdom of the crowd and handwriting recognition. 2012. CHI '12, 2895-2904.

10. Profita H. P., Clawson J., Gilliland S., Zeagler C., Starner T., Budd J., Do E. Y-L. 2013. Don't mind me touching my wrist. ISWC '13, 89-96.

11. Strachan S., Murray-Smith R., O'Modhrain S. 2007. BodySpace: inferring body pose for natural control of a music player. CHI'07 EA. 2001-2006. 


\begin{tabular}{|c|c|c|c|c|c|c|c|c|c|c|c|c|c|c|c|c|c|c|c|c|c|c|c|c|c|}
\hline a & b & c & d & e & $f$ & g & h & $\mathbf{i}$ & j & k & I & $\mathbf{m}$ & $\mathbf{n}$ & o & $\mathrm{p}$ & $q$ & $r$ & $\mathbf{s}$ & $t$ & $\mathrm{u}$ & $\mathrm{v}$ & w & $x$ & & lassified as \\
\hline 209 & 1 & 2 & 22 & 1 & 0 & 0 & 0 & 0 & 0 & 0 & 0 & 3 & 2 & 0 & 0 & 0 & 0 & 0 & 0 & 0 & 0 & 0 & 0 & $\mathbf{a}$ & head \\
\hline 1 & 234 & 0 & 3 & 0 & 0 & 0 & 0 & 0 & 0 & 0 & 0 & 1 & 0 & 1 & 0 & 0 & 0 & 0 & 0 & 0 & 0 & 0 & 0 & b & left ear \\
\hline 3 & 0 & 233 & 1 & 0 & 0 & 0 & 1 & 0 & 0 & 0 & 0 & 1 & 0 & 1 & 0 & 0 & 0 & 0 & 0 & 0 & 0 & 0 & 0 & c & right ear \\
\hline 20 & 1 & 2 & 211 & 0 & 1 & 1 & 0 & 0 & 0 & 0 & 0 & 4 & 0 & 0 & 0 & 0 & 0 & 0 & 0 & 0 & 0 & 0 & 0 & d & chin \\
\hline 2 & 0 & 0 & 0 & 201 & 5 & 4 & 0 & 1 & 1 & 0 & 0 & 23 & 1 & 2 & 0 & 0 & 0 & 0 & 0 & 0 & 0 & 0 & 0 & e & left shoulder \\
\hline 1 & 0 & 1 & 1 & 3 & 188 & 1 & 3 & 1 & 0 & 0 & 0 & 3 & 37 & 1 & 0 & 0 & 0 & 0 & 0 & 0 & 0 & 0 & 0 & $f$ & right shoulder \\
\hline 1 & 0 & 0 & 1 & 4 & 1 & 100 & 0 & 5 & 0 & 0 & 0 & 2 & 0 & 4 & 2 & 0 & 0 & 0 & 0 & 0 & 0 & 0 & 0 & $\mathbf{g}$ & left upper arm \\
\hline 0 & 0 & 0 & 0 & 0 & 2 & 0 & 107 & 0 & 4 & 0 & 1 & 1 & 2 & 1 & 2 & 0 & 0 & 0 & 0 & 0 & 0 & 0 & 0 & h & right upper arm \\
\hline 0 & 0 & 0 & 0 & 1 & 0 & 5 & 0 & 82 & 0 & 15 & 0 & 0 & 0 & 0 & 1 & 0 & 1 & 2 & 0 & 9 & 4 & 0 & 0 & $\mathrm{i}$ & left forearm \\
\hline 0 & 0 & 0 & 0 & 1 & 0 & 0 & 1 & 0 & 90 & 0 & 16 & 1 & 0 & 0 & 1 & 1 & 0 & 2 & 0 & 0 & 7 & 0 & 0 & $\mathbf{j}$ & right forearm \\
\hline 0 & 0 & 0 & 0 & 0 & 0 & 0 & 0 & 18 & 0 & 79 & 0 & 0 & 0 & 0 & 1 & 1 & 1 & 1 & 1 & 9 & 4 & 5 & 0 & $\mathbf{k}$ & left wrist \\
\hline 0 & 0 & 0 & 0 & 0 & 0 & 0 & 0 & 0 & 20 & 0 & 83 & 0 & 0 & 0 & 0 & 0 & 0 & 1 & 1 & 3 & 6 & 2 & 4 & I & right wrist \\
\hline 4 & 0 & 0 & 1 & 28 & 2 & 3 & 1 & 0 & 0 & 0 & 0 & 191 & 6 & 1 & 2 & 0 & 0 & 0 & 1 & 0 & 0 & 0 & 0 & m & left collarbone \\
\hline 0 & 1 & 0 & 1 & 2 & 28 & 1 & 5 & 0 & 0 & 0 & 0 & 9 & 193 & 0 & 0 & 0 & 0 & 0 & 0 & 0 & 0 & 0 & 0 & $\mathbf{n}$ & right collarbone \\
\hline 0 & 1 & 1 & 0 & 0 & 0 & 5 & 3 & 0 & 0 & 0 & 1 & 0 & 1 & 198 & 8 & 17 & 0 & 3 & 1 & 1 & 0 & 0 & 0 & o & left abdomen \\
\hline 0 & 0 & 0 & 0 & 0 & 0 & 2 & 3 & 2 & 0 & 1 & 0 & 0 & 0 & 5 & 179 & 1 & 36 & 1 & 8 & 2 & 0 & 0 & 0 & $\mathbf{p}$ & right abdomen \\
\hline 0 & 0 & 0 & 0 & 1 & 0 & 0 & 0 & 1 & 1 & 1 & 0 & 0 & 0 & 16 & 0 & 176 & 0 & 35 & 0 & 3 & 2 & 2 & 2 & $\mathbf{q}$ & left waist \\
\hline 0 & 0 & 0 & 0 & 0 & 0 & 0 & 0 & 0 & 1 & 0 & 0 & 0 & 0 & 0 & 29 & 1 & 173 & 1 & 31 & 1 & 2 & 0 & 1 & $r$ & right waist \\
\hline 0 & 0 & 0 & 0 & 0 & 0 & 1 & 0 & 2 & 0 & 1 & 2 & 0 & 0 & 1 & 1 & 31 & 0 & 180 & 0 & 17 & 3 & 1 & 0 & $\mathbf{s}$ & left pocket \\
\hline 0 & 0 & 0 & 0 & 0 & 0 & 0 & 0 & 1 & 3 & 2 & 1 & 0 & 0 & 0 & 1 & 0 & 29 & 5 & 177 & 3 & 15 & 0 & 3 & $\mathbf{t}$ & right pocket \\
\hline 0 & 0 & 0 & 0 & 0 & 0 & 0 & 0 & 13 & 0 & 7 & 5 & 0 & 0 & 0 & 0 & 1 & 0 & 14 & 2 & 161 & 14 & 16 & 7 & $\mathbf{u}$ & left thigh \\
\hline 0 & 0 & 0 & 0 & 0 & 0 & 0 & 0 & 4 & 9 & 5 & 4 & 0 & 0 & 0 & 1 & 2 & 1 & 2 & 12 & 17 & 156 & 7 & 20 & $\mathbf{v}$ & right thigh \\
\hline 0 & 0 & 0 & 0 & 0 & 0 & 0 & 0 & 1 & 0 & 6 & 4 & 0 & 0 & 0 & 0 & 1 & 0 & 2 & 4 & 17 & 11 & 152 & 42 & $w$ & left knee \\
\hline 0 & 0 & 0 & 0 & 0 & 0 & 0 & 0 & 0 & 3 & 0 & 1 & 0 & 0 & 0 & 0 & 0 & 0 & 1 & 7 & 13 & 20 & 50 & 145 & $\mathbf{x}$ & right knee \\
\hline
\end{tabular}

Figure 5. A confusion matrix based on the offline data set shows how different body parts might confuse each other. Several body locations have fewer instances, as they were out of reach half of the time, e.g., left forearm is unreachable for tapping with the left hand holding the phone. Note that confusion tended to happen when two user-defined locations are close to each other, e.g., collarbone vs. shoulder or waist vs. pant pocket.

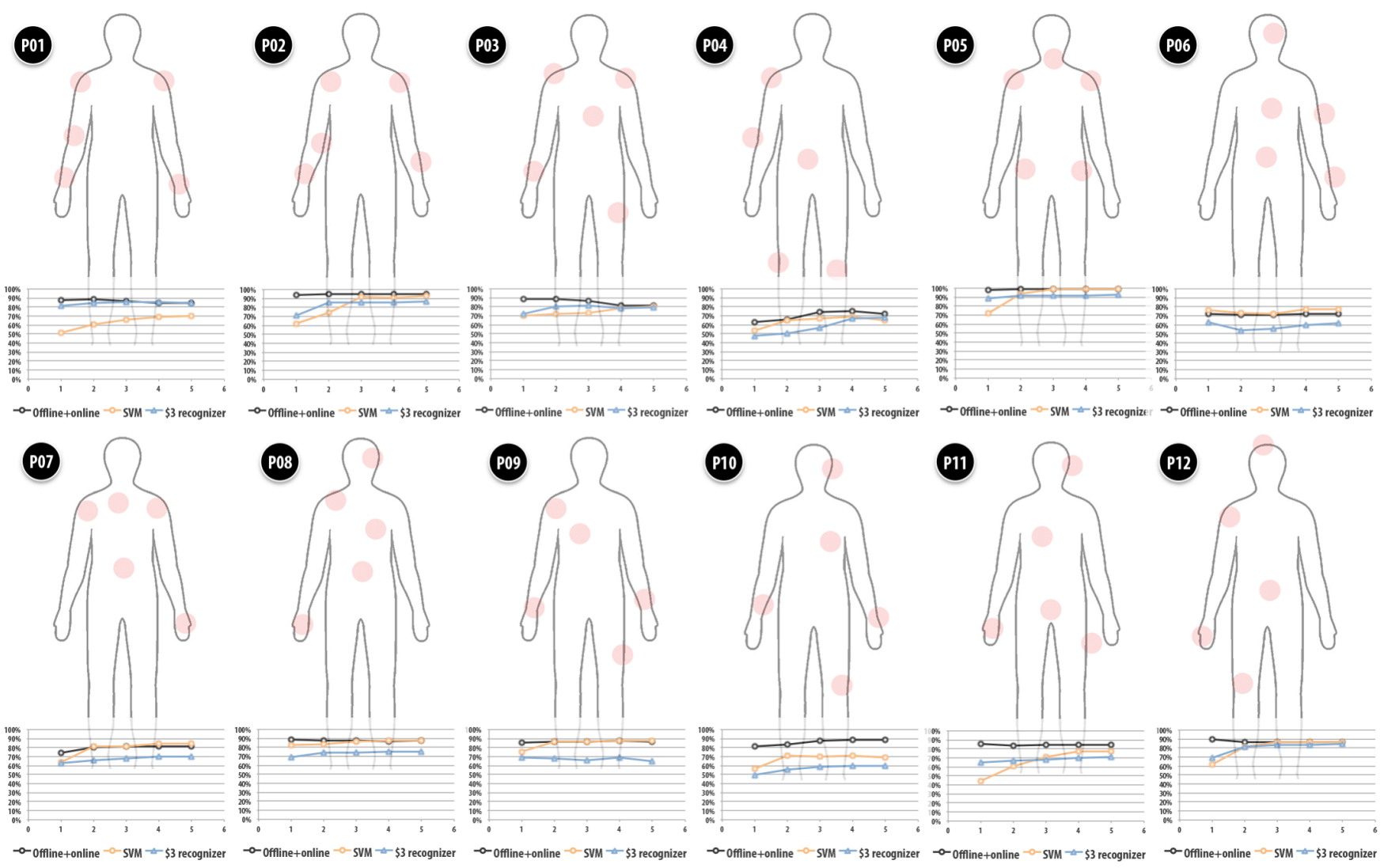

Figure 6. Each user's choice of tapping locations and the achieved accuracy across our approach and two baseline methods. 